Annals of Pure and Applied Mathematics

Vol. 16, No. 2, 2018, 437-441

ISSN: 2279-087X (P), 2279-0888(online)

Published on 24 March 2018

Annals of

www.researchmathsci.org

DOI: http://dx.doi.org/10.22457/apam.v16n2a21

Pure and Applied

Mathematics

\title{
On a Ramsey Problem Involving the 3-Pan Graph
}

\author{
Chula Jayawardene \\ Department of Mathematics, University of Colombo, Sri Lanka \\ Email: c_jayawardene@yahoo.com
}

Received 20 February 2018; accepted 21 March 2018

\begin{abstract}
Let $K_{\mathrm{s}}$ and $K_{j \times s}$ denote the complete graph on $s$ vertices and the complete multipartite balanced graph having $j$ partite sets (where $j \geq 3$ ) of size $s$ respectively. For any two graphs say $G, H$, we say that $K_{s} \rightarrow(H, G)$, if for any red/blue coloring of $K_{s}$, given by $K_{\mathrm{s}}=H_{R} \oplus H_{B}$, there exists a red copy of a $H$ in $H_{R}$ or a blue copy $G$ in $H_{B}$. In accordance with the same notation, we also say that $K_{j \times s} \rightarrow(H, G)$, if for any red/blue coloring of $K_{j \times s}$, given by $K_{j \times s}=H_{R} \oplus H_{B}$, there exists a red copy of a $H$ in $H_{R}$ or a blue copy $G$ in $H_{B}$. The balanced multipartite Ramsey number $m_{j}(G, H)$ is defined as the smallest positive number $s$ such that that $K_{j \times s} \rightarrow(H, G)$. There are 11 non-isomorphic graphs $G$ on 4 vertices, out of which 5 graphs $G$ are connected and the others are disconnected. In this paper we exhaustively find $m_{j}(P, G)$ for all of the 11 non-isomorphic graphs $G$ on 4 vertices where $P$ denotes the 3-pan graph (paw graph) given by $K_{l, 3}+e$.
\end{abstract}

Keywords: Graph theory, Ramsey theory

AMS Mathematics Subject Classification: 05C55, 05D10

\section{Introduction}

All graphs mentioned in this paper are simple graphs that do not contain loops or parallel edges. The diagonal classical Ramsey number $r(n, n)$, defined as the smallest positive integer $t$ such that $K_{t} \rightarrow\left(K_{n}, K_{n}\right)$, have been studied in detail and are known for almost all pairs of graphs when $n<5$. However, not much is known about the exact value of $r(5,5)$ other than that the upper bound is 48 (proved by Vigleik Angeltveit and Brendan D. McKay).

A new branch of the classical Ramsey numbers, namely the size Ramsey multipartite numbers $m_{j}(H, G)$, were introduced by Van Vuurenet al ([1]) and Baskoro et al([8]), a few decades ago. As of yet, the exact value $m_{j}(H, G)$, when $|V(G)|<5$, and $|V(H)|<5$ are known only for a few pairs of graphs. In this paper we exhaustively find the exact value of $m_{j}(P, G)$ for all of the 11 non-isomorphic graphs $G$ on 4 vertices, when $P$ is isomorphic to the 3-pan graph. 
Chula Jayawardene

The summary of our findings is illustrated in the following table.

\begin{tabular}{|c|c|c|c|c|c|c|c|c|c|}
\hline$m_{\mathrm{j}}\left(K_{1,3}+\mathrm{e}, G\right)$ & Graph $G$ & 3 & 4 & 5 & 6 & 7 & 8 & 9 & $\begin{array}{l}\text { Greater } \\
\text { than or } \\
\text { equal to } \\
10\end{array}$ \\
\hline Row 1 & $4 K_{l}$ & 2 & 1 & 1 & 1 & 1 & 1 & 1 & 1 \\
\hline Row 2 & $P_{2} \cup 2 K_{1}$ & 2 & 1 & 1 & 1 & 1 & 1 & 1 & 1 \\
\hline Row 3 & $2 K_{2}$ & 2 & 2 & 1 & 1 & 1 & 1 & 1 & 1 \\
\hline Row 4 & $P_{3} \cup K_{1}$ & 2 & 2 & 1 & 1 & 1 & 1 & 1 & 1 \\
\hline Row 5 & $P_{4}$ & 3 & 2 & 2 & 1 & 1 & 1 & 1 & 1 \\
\hline Row 6 & $K_{1,3}$ & 3 & 3 & 2 & 2 & 1 & 1 & 1 & 1 \\
\hline Row 7 & $C_{3} \cup K_{1}$ & $\infty$ & $\infty$ & $\infty$ & 2 & 1 & 1 & 1 & 1 \\
\hline Row 8 & $C_{4}$ & 3 & 2 & 2 & 2 & 1 & 1 & 1 & 1 \\
\hline Row 9 & $K_{1,3}+e$ & $\infty$ & $\infty$ & $\infty$ & 2 & 1 & 1 & 1 & 1 \\
\hline Row 10 & $B_{2}$ & $\infty$ & $\infty$ & $\infty$ & 2 & 1 & 1 & 1 & 1 \\
\hline Row 11 & $K_{4}$ & $\infty$ & $\infty$ & $\infty$ & $\infty$ & $\infty$ & $\infty$ & 2 & 1 \\
\hline
\end{tabular}

Table 1: Values of $m_{\mathrm{j}}\left(K_{1,3}+\mathrm{e}, G\right)$.

The next section deals with finding the entries of the above table. Clearly the rows corresponding to row 1 , row 2, row 4, row 5, row 6 and row 10 follows from Syafrizal and et al and Jayawardene et al (see $[3,4,5,6,8])$.

\section{Some useful lemmas on connected subgraphs of $K_{4}$}

Theorem 1. If $j \geq 3$, then

$$
m_{j}\left(K_{1,3}+e, C_{3}\right)= \begin{cases}1 & j \geq 7 \\ 2 & j=6 \\ \infty & j \in\{3,4,5\}\end{cases}
$$

Proof: If $j \geq 7$, since $r\left(K_{1,3}+\mathrm{e}, C_{3}\right)=7$ (see [2]), we get $m_{j}\left(K_{1,3}+\mathrm{e}, C_{3}\right)=1$.

Consider the graph $K_{6 \times 1}=H_{R} \oplus H_{B}$, such that $H_{R}$ equals to a $2 K_{3}$ and $H_{B}$ equals to a $K_{3,3}$. Then the graph has no red $K_{1,3}+\mathrm{e}$ and has no blue $C_{3}$. Therefore, $m_{6}\left(K_{1,3}+\mathrm{e}, C_{3}\right) \geq 2$. Next to show, $m_{6}\left(K_{1,3}+\mathrm{e}, C_{3}\right) \leq 2$ consider any red/blue coloring given by $K_{6 \times 2}=H_{R} \oplus H_{B}$, such that $H_{R}$ contains no red $K_{1,3}+$ eand $H_{B}$ contains no blue $C_{3}$. As $r\left(C_{3}, C_{3}\right)=6$ from [2] there is a red $C_{3}$, in $H_{R}$. Without loss of generality, assume that the red $C_{3}$, is induced by say $\mathrm{v}_{11}, \mathrm{v}_{21}, \mathrm{v}_{31}$. Let $S=\left\{v_{i 2} \mid i \in\{4,5,6\}\right\}$. Since $H_{R}$ contains no red $K_{1,3}+\mathrm{e}$, all edges joining $\mathrm{v}_{11}$ to each of the 6 elements in $S$ will be blue. If we consider the red/blue graphs generated by $S$, as $m_{3}\left(K_{1,3}+e, P_{2}\right)=2$, we get that it will contain a blue $P_{2}$. But then the 
On a Ramsey Problem Involving the 3-Pan Graph

vertices of this $P_{2}$ together with $\mathrm{v}_{11}$ will give us a blue $C_{3}$, a contradiction. Hence, $m_{6}\left(K_{1,3}+\mathrm{e}, C_{3}\right) \leq 2$. Therefore, $m_{6}\left(K_{1,3}+\mathrm{e}, C_{3}\right)=2$.

Finally, as $m_{i}\left(K_{1,3}+e, C_{3}\right) \geq m_{i}\left(C_{3}, C_{3}\right)$ for all $I$ and $m_{i}\left(C_{3}, C_{3}\right)=\infty$ for $j \in\{3, \ldots, 5\}$ (see [5]), we get that $m_{i}\left(K_{1,3}+e, C_{3}\right)=\infty$ for $j \in\{3, \ldots, 5\}$.

Theorem 2. If $j \geq 3$, then

$$
m_{j}\left(K_{1,3}+e, C_{4}\right)= \begin{cases}1 & j \geq 7 \\ 2 & j \in\{4,5,6\} \\ 3 & j=3\end{cases}
$$

Proof: Let $j \geq 3$. All values of $m_{j}\left(C_{4}, C_{3}\right)$ has been found in [5]. This gives us, $m_{j}\left(K_{1,3}+x\right.$, $C_{4}$ ) since $m_{j}\left(K_{1,3}+e, C_{4}\right)=m_{j}\left(C_{3}, C_{4}\right)$.

Theorem 3. If $j \geq 3$, then

$$
m_{j}\left(K_{1,3}+e, K_{1,3}+e\right)= \begin{cases}1 & j \geq 7 \\ 2 & j=6 \\ \infty & j \in\{3,4,5\}\end{cases}
$$

Proof: If $j \geq 7$, since $r\left(K_{1,3}+\mathrm{e}, K_{1,3}+e\right)=7$ (see [2]), we get $m_{j}\left(K_{1,3}+\mathrm{e}, K_{1,3}+\mathrm{e}\right)=1$.

Next color the graph $K_{6 \times 1}=H_{R} \oplus H_{B}$, such that $H_{R}=2 K_{3}$. Then the graph has no red $K_{1,3}+\mathrm{e}$ and has no blue $K_{1,3}+\mathrm{e}$. Therefore, $m_{6}\left(K_{1,3}, K_{1,3}+\mathrm{e}\right) \geq 2$. Next to show, $m_{6}\left(K_{1,3}+\mathrm{e}\right.$, $\left.K_{1,3}+\mathrm{e}\right) \leq 2$, consider any red/blue coloring given by $K_{6 \times 2}=H_{R} \oplus H_{B}$, such that $H_{R}$ contains no red $K_{1,3}+$ eand $H_{B}$ contains no blue $K_{1,3}+$ e. As $m_{6}\left(C_{3}, K_{1,3}+\right.$ e) $=2$ from [5] there is a red $C_{3}$, in $H_{R}$. Without loss of generality assume that the red $C_{3}$, is induced by say $\mathrm{v}_{11}, \mathrm{v}_{21}, \mathrm{v}_{31}$. But then if we consider the vertex $\mathrm{v}_{11}$ it must be adjacent in blue to all of the vertices of $\mathrm{v}_{41}, \mathrm{v}_{42}, \mathrm{v}_{52}, \mathrm{v}_{62}$ as otherwise would result in a red $K_{1,3}+\mathrm{e}$. But then all the edges $\left(\mathrm{v}_{41}, \mathrm{v}_{52}\right),\left(\mathrm{v}_{41}, \mathrm{v}_{62}\right),\left(\mathrm{v}_{42}, \mathrm{v}_{52}\right),\left(\mathrm{v}_{42}, \mathrm{v}_{62}\right)$ and $\left(\mathrm{v}_{52}, \mathrm{v}_{62}\right)$ will be forced to be red as otherwise it will result in a blue $K_{1,3}+\mathrm{e}$.

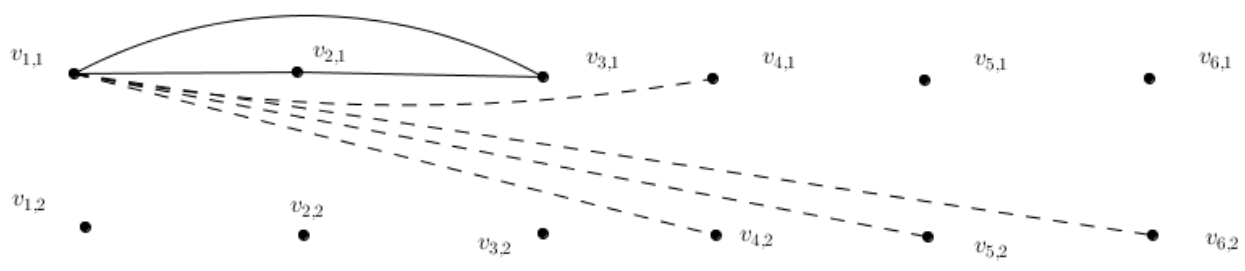

Figure 1: Diagram related to the proof of $m_{6}\left(K_{1,3}+\mathrm{e}, K_{1,3}+\mathrm{e}\right) \leq 2$ 


\section{Chula Jayawardene}

But then the vertex set $S=\left\{\mathrm{v}_{41}, \mathrm{v}_{42}, \mathrm{v}_{52}, \mathrm{v}_{62}\right\}$ will contain a red $K_{1,3}+\mathrm{e}$, a contradiction. Thus, $m_{6}\left(K_{1,3}+\mathrm{e}, K_{1,3}+\mathrm{e}\right) \leq 2$ Therefore, we get $m_{6}\left(K_{1,3}+e, K_{1,3}+e\right)=2$.

When $j \in\{3,4,5\}, m_{3}\left(C_{3}, K_{1,3}+e\right)=\infty$ follows from [5]. Therefore, as $\mathrm{C}_{3}$, is a subgraph $K_{1,3}+e$, it follows that, $m_{j}\left(K_{1,3}+\mathrm{e}, K_{1,3}+\mathrm{e}\right)=\infty$ for $j=\{3,4,5\}$, as required.

Theorem 4. If $j \geq 3$, then

$$
m_{j}\left(K_{1,3}+e, K_{4}\right)=\left\{\begin{array}{cc}
1 & j \geq 10 \\
2 & j=9 \\
\infty & j \in\{3, \ldots, 8\}
\end{array}\right.
$$

Proof: If $j \geq 10$, since $r\left(K_{1,3}+\mathrm{e}, K_{4}\right)=10$ (see [2]), we get $m_{j}\left(K_{1,3}+\mathrm{e}, K_{4}\right)=1$.

Consider the graph $K_{9 \times 1}=H_{R} \oplus H_{B}$, such that $H_{R}$ equals to a $3 K_{3}$ and $H_{B}$ equals to a $K_{3,3,3}$. Then the graph has no red $K_{1,3}+\mathrm{e}$ and has no blue $K_{4}$. Therefore, $m_{9}\left(K_{1,3}+\mathrm{e}, K_{4}\right) \geq 2$. Next to show, $m_{9}\left(K_{1,3}+\mathrm{e}, K_{4}\right) \leq 2$ consider any red/blue coloring given by $K_{9 \times 2}=H_{R} \oplus H_{B}$, such that $H_{R}$ contains no red $K_{1,3}+$ eand $H_{B}$ contains no blue $K_{4}$. As $r\left(C_{3}, K_{4}\right)=9$ from [2] there is a red $C_{3}$, in $H_{R}$. Without loss of generality assume that the red $C_{3}$, is induced by say $\mathrm{v}_{11}, \mathrm{v}_{21}, \mathrm{v}_{31}$. Let $S=\left\{v_{i 2} \mid i \in\{2,3, \ldots, 8\}\right\}$. Since $H_{R}$ contains no red $K_{1,3}+\mathrm{e}$, all edges joining $\mathrm{v}_{11}$ to each of the 7 elements in $S$ will be blue.If we consider the red/blue graphs generated by $S$, as $r\left(K_{1,3}+e, C_{3}\right)=7$, we get that it will contain a blue $C_{3}$. But then the vertices of this $C_{3}$ together with $\mathrm{v}_{11}$ will give us a blue $K_{4}$, a contradiction. Hence, $m_{9}\left(K_{1,3}+\mathrm{e}, K_{4}\right) \leq 2$. Therefore, $m_{9}\left(K_{1,3}+\mathrm{e}, K_{4}\right)=2$.

Finally, as $m_{i}\left(K_{1,3}+e, K_{4}\right) \geq m_{i}\left(C_{3}, K_{4}\right)$ for all $i$, and $m_{i}\left(C_{3}, K_{4}\right)=\infty$ for $j \in\{3, \ldots, 8\}$ (see [5]), we get that $m_{i}\left(K_{1,3}+e, K_{4}\right)=\infty$ for $j \in\{3, \ldots, 8\}$.

\section{Size Ramsey numbers $m_{j}\left(P_{4}, G\right)$ when $G$ is disconnected graph on 4 vertices}

We have already dealt with all cases excluding $G=2 K_{2}$. We will deal with this in the following theorem.

Theorem 5. If $j \geq 3$, then

$$
m_{j}\left(K_{1,3}+e, 2 K_{2}\right)= \begin{cases}2 & \text { if } j \in\{3,4\} \\ 1 & \text { if } j \geq 5\end{cases}
$$

Proof: Consider the coloring of $K_{4 \times 1}=H_{R} \oplus H_{B}$, generated by $H_{R}=K_{3}$. Then, $K_{4 \times 1}$ has no red $K_{1,3}+$ eor a blue $2 K_{2}$. Therefore, we obtain that $m_{4}\left(K_{1,3}+e, 2 K_{2}\right) \geq 2$. 
On a Ramsey Problem Involving the 3-Pan Graph

To show $m_{3}\left(K_{1,3}+e, 2 K_{2}\right) \leq 2$, consider any red/blue coloring given by $K_{3 \times 2}=H_{R}$ $\bigoplus H_{B}$, such that $H_{R}$ contains no red $K_{1,3}+$ eand $H_{B}$ contains no blue $2 K_{2}$. Since $H_{R}$ contains no red $K_{1,3}+e$ without loss of generality we may assume that there is at least one blue edge in $K_{3 \times 2}$ say $\left(v_{11}, v_{21}\right)$. Next as there is no blue $2 K_{2}$ all edges not adjacent to $\left(v_{11}, v_{21}\right)$ in $K_{3 \times 2}$ must be red. Thus, in particular $\left(v_{12}, v_{22}\right),\left(v_{12}, v_{31}\right),\left(v_{12}, v_{32}\right)$ and $\left(v_{22}, v_{31}\right)$ must be red edges. Thus, we get a red $K_{1,3}+e$, a contradiction. That is, $m_{3}\left(K_{1,3}+e, 2 K_{2}\right) \leq 2$. Therefore, $m_{3}\left(K_{1,3}, 2 K_{2}\right)=2$ and $m_{4}\left(K_{1,3}, 2 K_{2}\right)=2$. Finally, $m_{j}\left(K_{1,3}+e, 2 K_{2}\right)=1$ when $j \geq 5$, as $r\left(K_{1,3}+e, 2 K_{2}\right)=5$ (see [2]).

\section{REFERENCES}

1. A.P.Burger and J.H.Van Vuuren, Ramsey numbers in complete balanced multipartite graphs. Part II: size numbers, Discrete Math., 283 (2004) 45-49.

2. V.Chv'atal and F.Harary, Generalized Ramsey theory for graphs, III. Small off diagonal numbers, Pacific Journal of Mathematics, 41(2) (1972) 335-345.

3. M.Christou, S.Iliopoulos and M.Miller, Bipartite Ramsey numbers involving stars, stripes and trees, Electronic Journal of Graph Theory and Applications, 1(2) (2013) 89-99.

4. C.J.Jayawardene and L.Samerasekara, Size Multipartite Ramsey numbers for $K_{4}$ eversus all graphs $G$ up to 4 vertices, Annals of Pure and Applied Mathematics, 13(1) (2017) 9-26.

5. C.J.Jayawardene and L.Samerasekara, Size Ramsey numbers for $C_{3}$ versus all graphs $G$ up to 4 vertices, National Science Foundation, 45(11) (2017) 67-72.

6. V.Kavitha and R.Govindarajan, A study on Ramsey numbers and its bounds, Annals of Pure and Applied Mathematics, 8(2) (2014) 227-236.

7. M.S.Sunitha and S.Mathew, Fuzzy graph theory: a survey, Annals of Pure and Applied Mathematics, 4(1) (2013) 92-110.

8. S.Sy, E.T.Baskoro and S.Uttunggadewa, The size multipartite Ramsey number for paths, Journal Combin. Math. Combin. Comput., 55 (2005) 103-107. 